University of Wollongong

Research Online

Faculty of Engineering and Information

Faculty of Engineering and Information

Sciences - Papers: Part A

Sciences

$1-1-2012$

\title{
Segmentations of through-the-wall radar images
}

C H. Seng

University of Wollongong, aseng@uow.edu.au

M G. Amin

Villanova University, mamin@uow.edu.au

F Ahmad

Villanova University

A Bouzerdoum

University of Wollongong, bouzer@uow.edu.au

Follow this and additional works at: https://ro.uow.edu.au/eispapers

Part of the Engineering Commons, and the Science and Technology Studies Commons

Research Online is the open access institutional repository for the University of Wollongong. For further information contact the UOW Library: research-pubs@uow.edu.au 


\title{
Segmentations of through-the-wall radar images
}

\author{
Abstract \\ In this paper, we examine the use of image segmentation approaches for target detection in TWRI. The \\ betweenclass variance thresholding, entropy-based segmentation, and Kmeans clustering are applied to \\ segment target and clutter regions. Real 2D polarimetric images are used to demonstrate that simple \\ histogram-based segmentation methods produce either comparable or improved performance over the \\ Likelihood Ratio Tests (LRT) detector. Specifically, the results show that, for the cases considered, the \\ entropy-based segmentation outperforms the other image segmentation methods and the LRT detector.

\section{Keywords} \\ wall, images, segmentations, radar \\ Disciplines \\ Engineering | Science and Technology Studies

\section{Publication Details} \\ C. H. Seng, M. G. Amin, F. Ahmad \& A. Bouzerdoum, "Segmentations of through-the-wall radar images," in \\ 2012 IEEE Radar Conference: Ubiquitous Radar, RADARCON 2012, 2012, pp. 647-652.
}




\title{
Segmentations of Through-the-Wall Radar Images
}

\author{
C. H. Seng*, M. G. Amin ${ }^{\dagger}$, F. Ahmad ${ }^{\dagger}$, and A. Bouzerdoum* \\ *School of Electrical, Computer and Telecommunications Engineering, University of Wollongong, \\ Wollongong, NSW 2522, Australia. \\ Email: \{aseng, a.bouzerdoum\}@uow.edu.au \\ ${ }^{\dagger}$ Radar Imaging Lab, Center for Advanced Communications, Villanova University, \\ Villanova, PA 19085, USA. \\ Email: \{moeness.amin, fauzia.ahmad\}@villanova.edu
}

\begin{abstract}
In this paper, we examine the use of image segmentation approaches for target detection in TWRI. The betweenclass variance thresholding, entropy-based segmentation, and Kmeans clustering are applied to segment target and clutter regions. Real 2D polarimetric images are used to demonstrate that simple histogram-based segmentation methods produce either comparable or improved performance over the Likelihood Ratio Tests (LRT) detector. Specifically, the results show that, for the cases considered, the entropy-based segmentation outperforms the other image segmentation methods and the LRT detector.
\end{abstract}

\section{INTRODUCTION}

Imaging of building interiors has recently been a subject of interest in many applications related to rescue missions, homeland security, and defense [1]. Indoor images are typically characterized by the presence of both spatially extended targets, like exterior and interior walls, and compact targets, such as humans. Also, near-field operations give rise to point spread functions that vary in range and cross-range [2]. Accordingly, the same target can have different spatial distribution, depending on its position.

The operator of the radar imaging system is faced with the difficult task of separating the target pixels from those of noise and clutter. Original images produced by backprojetion methods are often cluttered by the second and higher order reflections including targets and walls and by antenna cross talk. This requires the application of image segmentation techniques to distinguish between target and non-target regions in the image.

Detection of stationary targets in through-the-wall radar imaging (TWRI) using statistical detectors based on likelihood ratio tests (LRT) applied to the image pixels has been discussed in [3], [4]. Specifically, a Neyman-Pearson (NP) test was used to detect targets in indoor radar images by defining pixel-wise null and alternative hypotheses, coupled with a user-defined false alarm rate (FAR). The detector then generates a binary mask that depicts the target locations in the image as an output. However, the LRT approach also assumes particular probability density functions (PDFs) with a

\footnotetext{
* The work by C.H. Seng and A. Bouzerdoum has been supported in part by the Australian Research Council.

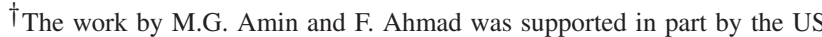
ARL contract W911NF-07-D-0001 and by ONR grant N0014-10-1-0455.
}

set of unknown test parameters. The need to define appropriate PDFs for target and clutter in the image, and to specify an appropriate FAR by the operator presents a shortcoming of the LRT detector, since in most cases, neither the PDFs nor the FAR is known a priori.

In this paper, rather than setting the threshold using LRT, we use image segmentation methods for target detection in TWRI. Segmentation of radar images is often performed with the ultimate goal to improve the image quality for subsequent analysis and scene description [5]. Generally, radar image segmentation is essential for applications such as target detection, classification, and identification. Hence, we investigate image segmentation methods for TWRI, which can also be applied for target detection. There have been a number of survey papers on thresholding [6], [7]. We cannot possibly apply and compare all existing thresholding methods in this paper. Yet, we chose three commonly used image segmentation methods for evaluation and comparison, namely, the between-class variance (BCV) thresholding [8], entropybased segmentation [9], and K-means clustering [10]. All of the above methods are object independent and expected to cast strong target and clutter as objects. Thus, walls, together with the targets, are expected to be highlighted. The segmentation process associated with these techniques produces threshold values, thus generating binary masks that are similar to that of the LRT detector. It is noted that image segmentation methods have been applied for object detection in Synthetic Aperture Radar (SAR) aerial images and Ground Penetrating Radar (GPR) images [5], [11]. They have also been considered as a pre-processing stage for change detection and object classification [12], [13] in radar images.

The LRT detector and the proposed segmentation methods are evaluated and compared using real two-dimensional (2D) polarimetric images collected at the Radar Imaging Lab, Villanova University. Since both methods produce a binary mask, their performance is assessed in terms of improvement in separation of target from clutter in individual images. We show that, for the cases considered, the entropy-based segmentation method outperforms other image segmentation techniques, including the LRT detector, by providing higher clutter suppression, while successfully maintaining the target regions. The $\mathrm{BCV}$ thresholding and $\mathrm{K}$-means clustering 
methods provide similar results to those of the entropybased segmentation when considering target regions; however, their respective clutter levels are much higher. Nevertheless, the proposed methods are more advantageous than the LRT detector since they do not require a predefined PDF and FAR.

The remainder of the paper is organized as follows Section II reviews the LRT target detection method proposed in [3], [4]. The image segmentation methods, which form an alternative to through-the-wall radar target detection is then described in Section III. Section IV evaluates the performance of the LRT detector and image segmentation methods using real data, and Section V concludes the paper.

\section{Likelihood Ratio Tests Detector}

In this section, we review the general image domain framework for LRT based target detection. Specifically, we consider the iterative version of the pixel-wise NP detector introduced in [3] that adapts the test parameters to the radar image statistics.

Let $\breve{I}_{j}, j=1,2, \ldots, J$ be the set of $J$ magnitude images. The pixel-wise NP test is given as

$$
\prod_{j=1}^{J} \frac{P_{r}\left(\breve{I}_{j} \mid H_{1}\right)}{P_{r}\left(\breve{I}_{j} \mid H_{0}\right)} \gtrless \gamma,
$$

where $H_{0}$ and $H_{1}$ denote, respectively, the null (target absent) and alternative (target present) hypothesis. The functions $P_{r}\left(\cdot \mid H_{0}\right)$ and $P_{r}\left(\cdot \mid H_{1}\right)$ are the conditional PDFs under the null and alternative hypothesis, respectively, and the parameter $\gamma$ is the likelihood ratio threshold, which can be obtained by specifying a desired FAR, $\alpha$, as

$$
\alpha=\int_{\gamma}^{\infty} P_{\ell}\left(\ell \mid H_{0}\right) d \ell
$$

where $P_{\ell}\left(\ell \mid H_{0}\right)$ denotes the distribution of the likelihood ratio under the null hypothesis.

Let $\hat{\theta}_{H_{0}}^{0}$ and $\hat{\theta}_{H_{1}}^{0}$ denote the initial estimates of the parameter vectors $\theta_{H_{0}}$ and $\theta_{H_{1}}$ describing the PDFs under $H_{0}$ and $H_{1}$, respectively. Given a FAR $\alpha$, a binary image $B_{N P}^{1}$, where superscript 1 represents the first iteration, can be obtained by evaluating (1). In order to enhance and optimize the estimation of the noise and test PDF parameters, morphological filtering is employed to obtain the binary image $B_{M F}^{1}$ (see [4] for details). This image can be used as a mask on the original set of images in order to obtain the revised parameter estimates $\hat{\theta}_{H_{0}}^{1}$ and $\hat{\theta}_{H_{1}}^{1}$. These revised parameters are then fed back to the NP test to obtain an improved detection result. The iteration stops when convergence is achieved.

It is noted that the final output of the LRT detector described above is a single binary image that indicates the presence of the targets.

\section{IMAgE SEgmentation}

Image segmentation based on histogram information is a simple technique which involves the basic assumption that the objects and the background in the sensed image have distinct gray level distributions. Since objects in remotely sensed imagery are often homogeneous, threshold values separating two or more distinct peaks in the gray level histogram can be obtained. Threshold selection methods can be classified into two groups, namely, global methods and local methods. A global thresholding technique separates the entire image into target and background regions with a single threshold value, whereas local thresholding methods partition the given image into a number of sub-images and determine a threshold for each of the sub-images separately. As global thresholding methods are computationally less intensive, they have been more popular for radar image analysis [14]. In this paper, we consider two of the commonly applied global thresholding methods, namely, between-class variance (BCV) thresholding [8] and entropy-based segmentation [9]. Since image segmentation can also be viewed as the partitioning of the observed intensities into groups, we also consider the application of $\mathrm{K}$ means clustering [10].

Consider the histogram of an input image as a discrete PDF $\rho(i)$ :

$$
\rho(i)=f_{i} / N
$$

with $\rho(i) \geq 0$ and $\sum_{i=0}^{L-1} \rho(i)=1$, where $f_{i}$ is the frequency of intensity level $i$ and $N$ is the total number of pixels in the image. Each pixel in the image assumes an intensity level from the set $\{0,1, \ldots, L-1\}$, where $L$ denotes the number of intensity levels or histogram bins.

\section{A. Between-Class Variance Thresholding}

The BCV thresholding method segments an image into two near-uniform regions by maximizing the sum of class variances [8]:

$$
\begin{aligned}
f_{B C V} & =\arg \max _{d}\left\{p_{r 1}(d)\left[m_{r 1}(d)-m_{\breve{I}}\right]^{2}\right. \\
& \left.+p_{r 2}(d)\left[m_{r 2}(d)-m_{\breve{I}}\right]^{2}\right\},
\end{aligned}
$$

where $m_{\breve{I}}$ is the mean image intensity. By dividing the histogram into two regions with intensity level $d$, the respective region probabilities can be expressed as

$$
p_{r 1}(d)=\sum_{i=0}^{d} \rho(i), \quad p_{r 2}(d)=\sum_{i=d+1}^{L} \rho(i) .
$$

Similarly, the means of the respective regions are given as

$$
m_{r 1}(d)=\sum_{i=0}^{d} \frac{i \cdot \rho(i)}{p_{r 1}(d)}, \quad m_{r 2}(d)=\sum_{i=d+1}^{L} \frac{i \cdot \rho(i)}{p_{r 2}(d)} .
$$

All values of $d=1,2, \ldots, L-2$ are considered and the corresponding functions $(4-6)$ are evaluated. The intensity value, $d$, that produces the maximum sum of the class variances is chosen as the threshold value $T_{B C V}$.

\section{B. Entropy-Based Segmentation}

Similar to the BCV method, the entropy-based segmentation decides on the threshold value in an exhaustive fashion. Instead of maximizing the sum of class variances, the entropy-based segmentation maximizes the sum of class entropies. Based on 
the information derived from the image histogram, the entropy of two regions is maximized using the following equation [9]:

$$
f_{H}=\arg \max _{d}\left\{H_{r 1}(d)+H_{r 2}(d)\right\},
$$

where $H_{r 1}(\cdot)$ and $H_{r 2}(\cdot)$ are the respective region entropies. Let $p_{i}$ be the probability of intensity level $i$ and $P_{d}=\sum_{i=1}^{d} p_{i}$ is the total probability. The entropy of each region can be expressed as

$$
\begin{aligned}
& H_{r 1}(d)=-\sum_{i=1}^{d} \frac{p_{i}}{P_{d}} \ln \frac{p_{i}}{P_{d}} \\
& H_{r 2}(d)=-\sum_{i=d+1}^{L} \frac{p_{i}}{P_{d}} \ln \frac{p_{i}}{P_{d}} .
\end{aligned}
$$

Given that the entropy for a region can also be calculated as

$$
H_{d}=-\sum_{i=1}^{d} \rho(i) \ln \rho(i),
$$

the total entropy of the image can be expressed as

$$
H_{\text {tot }}=-\sum_{i=1}^{L} \rho(i) \ln \rho(i) .
$$

Thus, (8 - 9) can be simplified as follows:

$$
\begin{aligned}
H_{r 1}(d) & =-\sum_{i=1}^{d} \frac{p_{i}}{P_{d}} \ln \frac{p_{i}}{P_{d}} \\
& =\ln \left(P_{d}\right)+\frac{H_{d}}{P_{d}} \\
H_{r 2}(d) & =-\sum_{i=d+1}^{L} \frac{p_{i}}{P_{d}} \ln \frac{p_{i}}{P_{d}} \\
& =\ln \left(1-P_{d}\right)+\frac{H_{t o t}-H_{d}}{1-P_{d}}
\end{aligned}
$$

Evaluating $d$ from 1 to $L-2$, the intensity value, $d$, that produces the maximum sum of the distribution entropies is chosen as the threshold value $T_{H}$.

\section{K-Means Clustering}

Clustering methods partition the observed intensities into classes, which are generally unknown and are explored based on the data by using a similarity measure. Given $N$ pixels, the $\mathrm{K}$-means clustering method partitions them into $K$ clusters by minimizing the sum of the within-cluster variances (WCSS) [10]:

$$
W C S S=\sum_{k=1}^{K} \sum_{i=1}^{N}\left\|v_{i}^{k}-\mu_{k}\right\|^{2},
$$

where $v_{i}^{k}$ is the $i$-th sample of the $k$-th class with centroid $\mu_{k}$. The pseudo-code for the K-means clustering is given as follows:

1) Initialize the number of classes $K$ and centroids $\mu_{k}$.

2) Assign each pixel to the group whose centroid is the closest.
3) After all the pixels have been assigned, re-calculate the centroids.

4) Repeat Steps 2 and 3 until the centroids no longer change.

Although the K-means is computationally very efficient, the major disadvantage is the need to specify the number of classes a priori. In the absence of this knowledge, one may resort to measures that could estimate the number of classes automatically [15]. This approach is not considered here. Instead, we set the number of classes as two to ensure consistency with the other image segmentation methods.

\section{EXPERIMENTAL RESULTS}

We evaluate both the LRT detector and the image segmentation methods using real 2D polarimetric images collected at the Radar Imaging Lab, Villanova University. The scene consists of calibrated targets, acquired from a single viewpoint using multiple polarizations. Both co-polarization $(\mathrm{HH}$ and $\mathrm{VV}$ ) and cross-polarization (HV) data sets were collected from the scene, containing a sphere, a top hat, a vertical dihedral, two dihedrals rotated at 22.5 and 45 degrees, respectively, and two trihedrals, all placed at different downranges, cross-ranges, and elevations, as shown in Fig. 1. For each polarization setting, the scene is imaged with a $1 \mathrm{GHz}$ waveform centered at $2.5 \mathrm{GHz}$ through a non-homogeneous plywood and gypsum board wall using a 57-element linear array with an interelement spacing of $22 \mathrm{~mm}$. The input images obtained from the scene are shown in Fig. 2. It can be observed that only two targets are visible in the cross-polarization image. This is due to the fact that the rotated dihedrals produce a stronger cross-polarization return.

We note that all radar images presented in this Section are plotted on a $35 \mathrm{~dB} \log$ scale, and the vertical and the horizontal axes represent the downrange and cross-range, respectively, with units in meters. The FAR for the LRT detector was fixed at $2.5 \%$.

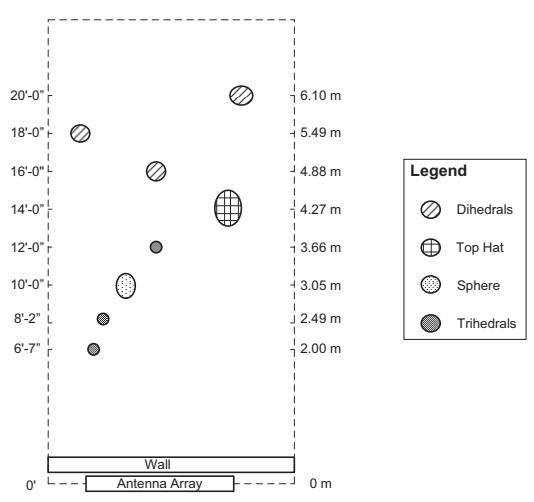

Fig. 1. Schematic of the scene.

After applying the LRT detector and image segmentation methods to the input images, the generated binary images are then used as a mask on the original images to produce corresponding enhanced images. The image enhancements are 

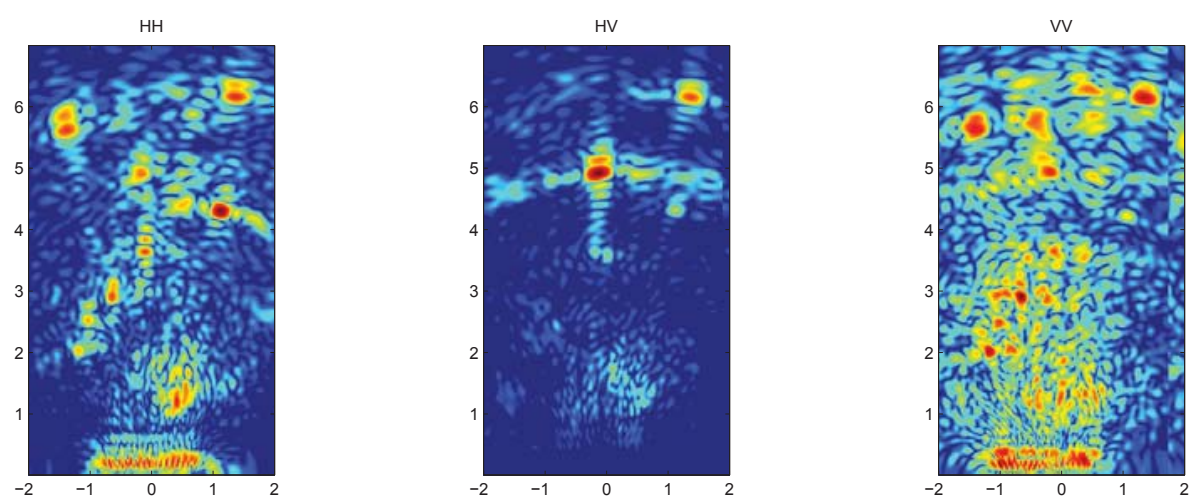

Fig. 2. Images acquired from the scene.

compared using the Improvement Factor of the Target-toClutter Ratio, denoted as IF. Let $\mathcal{P}_{\mathcal{R}, \breve{I}}$ denotes the average power of region $\mathcal{R}$ in image $\breve{I}$. The IF is given as

$$
\text { IF }=10 \log _{10}\left[\frac{\mathcal{P}_{\mathcal{R}_{t}, \breve{I_{e}}} \times \mathcal{P}_{\mathcal{R}_{c}, \breve{I}_{i}}}{\mathcal{P}_{\mathcal{R}_{t}, \breve{I_{i}}} \times \mathcal{P}_{\mathcal{R}_{c}, \breve{I_{e}}}}\right],
$$

where $\breve{I}_{i}$ is the input image and $\breve{I}_{e}$ is the enhanced image. $\mathcal{P}_{\mathcal{R}_{r}, \breve{I}_{m}}$ can be expressed as

$$
\mathcal{P}_{\mathcal{R}_{r}, \breve{I}_{m}}=\frac{1}{N_{\mathcal{R}_{r}}} \sum_{x_{q} \in \mathcal{R}_{r}}\left(\breve{I}_{m}\left(x_{q}\right)\right)^{2},
$$

where $\breve{I}_{m}\left(\mathbf{x}_{q}\right)$ is the $q$-th pixel of region $\mathcal{R}_{r}$ in image $\breve{I}_{m}$, and $N_{\mathcal{R}_{t}}$ and $N_{\mathcal{R}_{c}}$ are the number of pixels in the target region, $\mathcal{R}_{t}$, and clutter region, $\mathcal{R}_{c}$, respectively.

The results of applying both methods to the input images in Fig. 2 are presented in Fig. 3. We observe that the LRT detector applied to the individual images did not yield image enhancements. The noise and clutter present in the original images persist even after application of the LRT detector As for the BCV thresholding, the clutter has been slightly reduced. The K-means clustering produces a similar result to that of the BCV. Although there were some missed detections, visually, the entropy-based segmentation outperforms all the other methods by producing images with low clutter levels.

The IFs for the results are provided in Table I. With the exception of the cross-polarization case, where the BCV thresholding and the K-means clustering removed all the clutter to obtain the highest IF, it is evident that the entropybased segmentation generally outperforms the other methods by producing enhanced images with high IF; albeit with some missed detections.

TABLE I

IF IN TARGET-TO-CLUTTER RATIO AFTER IMAGE ENHANCEMENTS

\begin{tabular}{|l|c|c|c|}
\hline & HH & HV & VV \\
\hline LRT Detector 2.5\% FAR & 1.962 & 2.5124 & 3.0411 \\
\hline BCV Thresholding & 2.5501 & $\mathbf{1 7 . 7 0 6 3}$ & 2.1898 \\
\hline Entropy-based Segmentation & $\mathbf{4 . 3 5 1 6}$ & 16.2246 & $\mathbf{6 . 3 9 7 9}$ \\
\hline K-Means Clustering & 2.571 & $\mathbf{1 7 . 7 0 6 3}$ & 2.2344 \\
\hline
\end{tabular}

From the experimental results, it can be observed that the BCV thresholding and K-means clustering have similar performances and their differences in the IF are almost negligible. This is due to the fact that the K-means clustering's minimization of the WCSS is equivalent to the BCV thresholding's maximization of the sum of class variances when there are only two regions [8]. It can also be observed that the $\mathrm{BCV}$ thresholding generally has a poorer performance than the entropy-based segmentation. This is because the sparsity of the high pixel values (target pixels) in a through-the-wall radar image requires the $\mathrm{BCV}$ thresholding method to also include low pixel values (clutter pixels) in its class, in order to obtain a high class variance. This is evident from Fig. 4 that the maximum class variances for both regions in the copolarization and cross-polarization images are skewed towards the lower pixel regions.

Since the maximum class variances for the second region in the co-polarization images are also located in the lower pixel values region, the summation of both class variances produce a threshold value that is also located in the lower pixel values region. As a result, the segmented co-polarization image included most of the clutter. On the other hand, since the maximum class variance for the second region of the crosspolarization image is located at a much higher pixel value, its dominance causes the summation of both class variances to produces a much higher threshold value.

As for the entropy-based segmentation, it can be observed from Fig. 5 that the respective class entropies are less affected by the skewness towards the low pixel values region. As the threshold value is increased, the entropy of one region will generally decrease while the entropy of the other region will increase. Thus, the maximization of the sum of class entropies will produce a balanced threshold where the information content in both regions are almost equal.

\section{CONCLUSION}

In this paper, we examined image processing approaches for target detection in TWRI. Image segmentation techniques are applied to enhance the images by distinguishing between the target and clutter regions. Performance of the various 

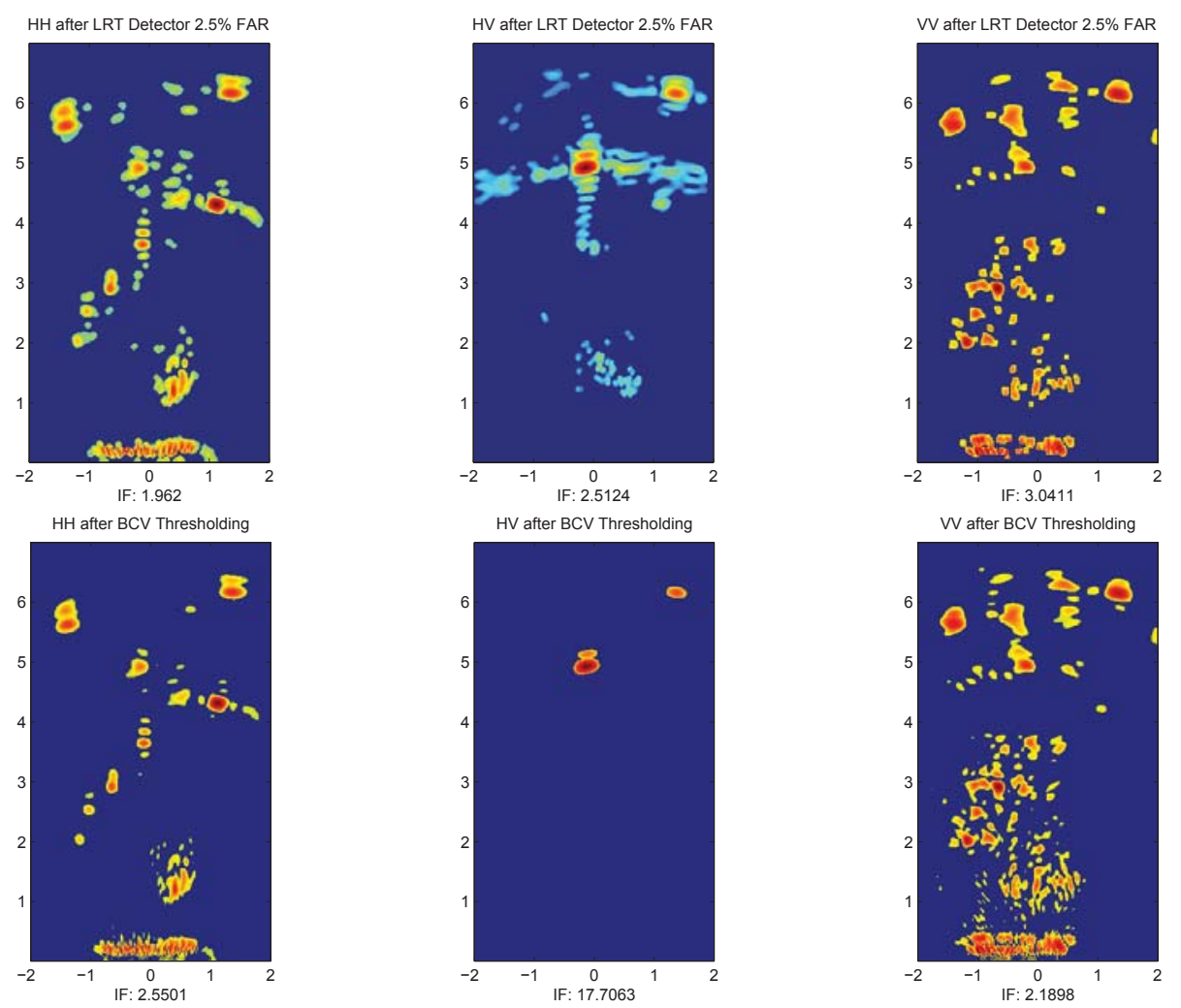

HV after BCV Thresholding

VV after BCV Thresholding
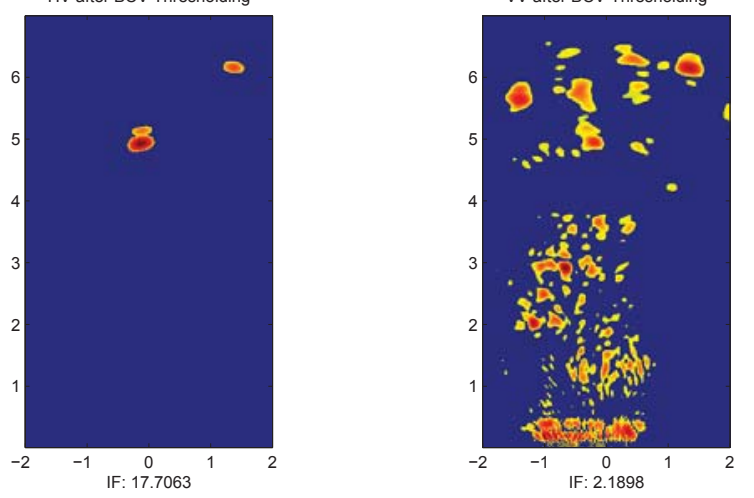

HH after Entropy Segmentation
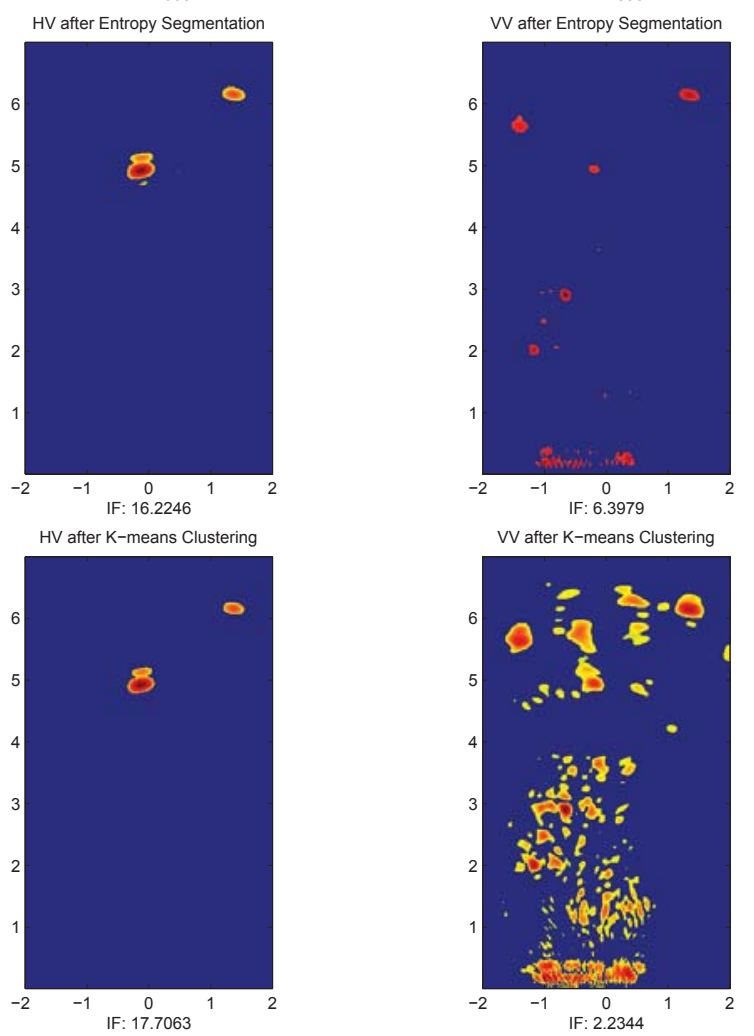

Fig. 3. Target detection and segmentation: from top to bottom, LRT detector, BCV thresholding, entropy-based segmentation, and K-means clustering. 

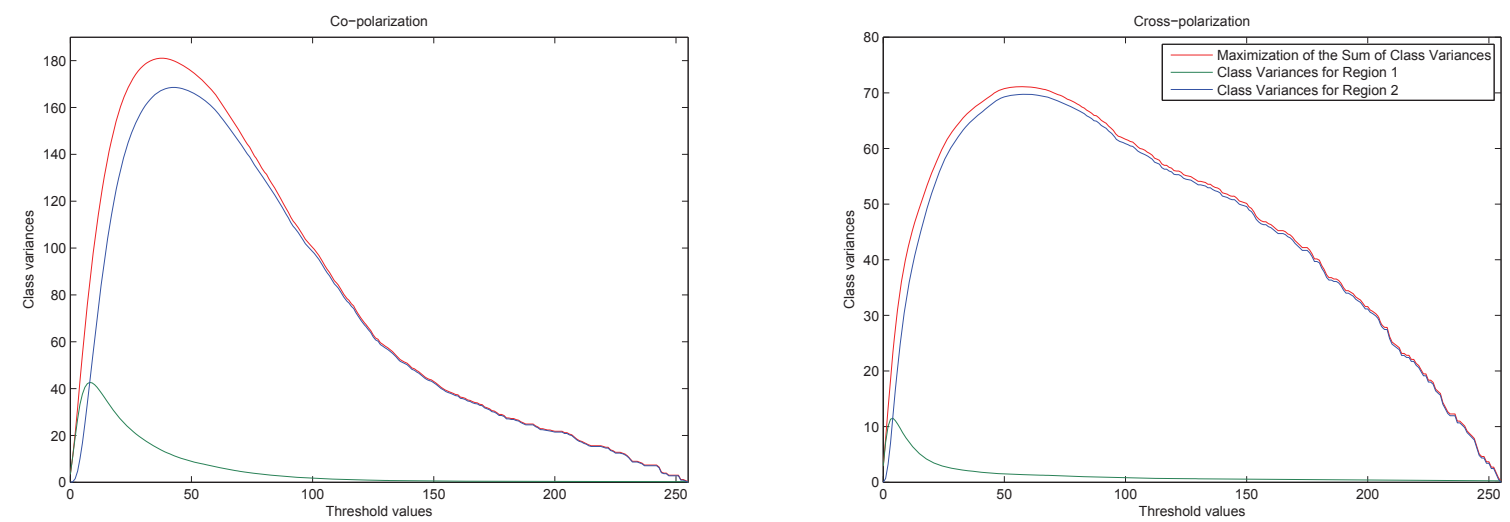

Fig. 4. Class variances with respect to the threshold values for co-polarization images (right) and cross-polarization image (left).
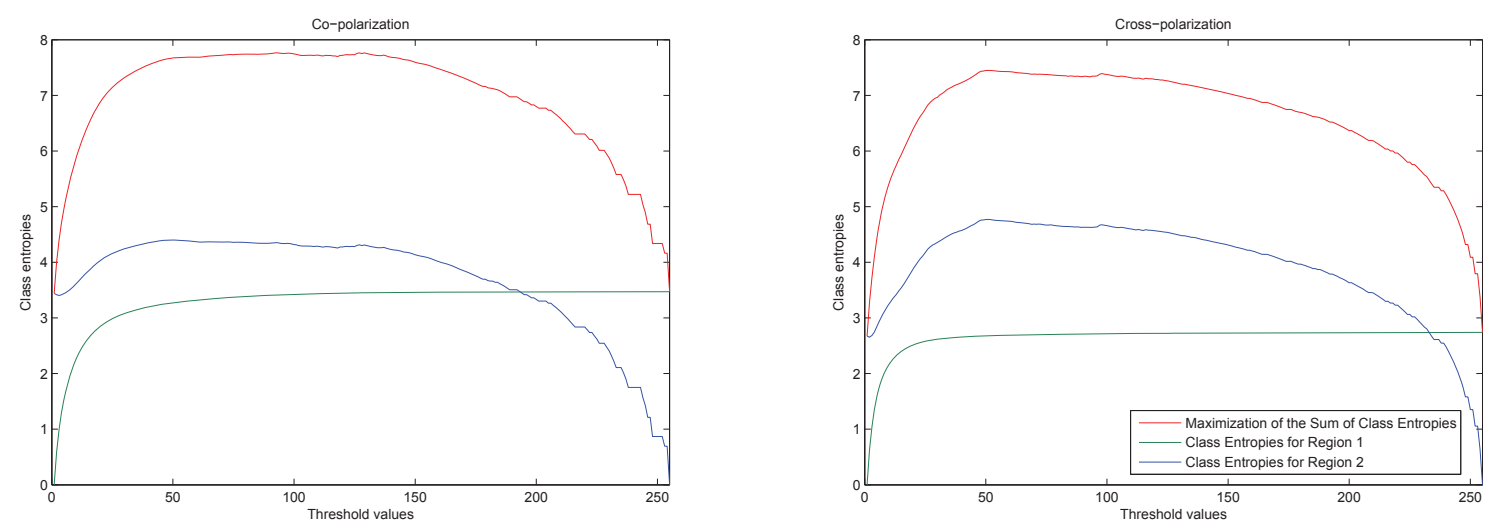

Fig. 5. Class entropies with respect to the threshold values for co-polarization images (right) and cross-polarization image (left).

approaches were evaluated using real 2D polarimetric images. The results showed that the entropy-based segmentation method outperforms the other segmentation schemes, as well as the LRT detector. Although there were some missed detections, the entropy-based segmentation consistently provided high target and low clutter levels. While the BCV thresholding and K-means clustering also maintained most of the targets, the clutter levels were much higher by comparison. Compared to the LRT detector, in addition to enhanced performance, the image segmentation techniques do not require pre-set values and are free from human intervention. Hence, for the cases considered, the entropy-based segmentation is most effective and viable alternative for target detection in TWRI.

\section{REFERENCES}

[1] M. Amin, Through the Wall Radar Imaging. CRC Press, 2010.

[2] F. Ahmad and M. Amin, "Multi-location wideband synthetic aperture imaging for urban sensing applications," Journal of the Franklin Institute, vol. 345, no. 6, pp. 618-639, 2008.

[3] C. Debes, M. Amin, and A. Zoubir, "Target detection in single- and multiple-view through-the-wall radar imaging," IEEE Trans. Geosci. Remote Sens., vol. 47, no. 5, pp. 1349-1361, 2009.

[4] C. Debes, J. Riedler, A. Zoubir, and M. Amin, "Adaptive target detection with application to through-the-wall radar imaging," IEEE Trans. Signal Process., vol. 58, no. 11, pp. 5572-5583, 2010.
[5] J.-S. Lee and I. Jurkevich, "Segmentation of SAR images," IEEE Trans. Geosci. Remote Sens., vol. 27, no. 6, pp. 674-680, 1989.

[6] R. Schoenmakers and L. Vuurpijl, "Segmentation and classification of combined optical and radar imagery," in Proc. Int. Geosci. Remote Sens. Symp., 1995, pp. 2151-2153.

[7] M. Sezgin and B. Sankur, "Survey over image thresholding techniques and quantitative performance evaluation," J. of Electronic Imag., vol. 13, no. 1 , pp. 146-165, 2004.

[8] N. Otsu, "A threshold selection method from gray-level histogram," IEEE Trans. Sys., Man. Cybern., vol. 9, no. 1, pp. 62-66, 1979.

[9] J. Kapur, P. Sahoo, and A. Wong, "A new method for gray-level picture thresholding using the entropy of the histogram," Comput. Vision, Graphics, and Image Process., vol. 29, no. 3, pp. 273-285, 1985.

[10] G. Seber, Multivariate Observations. John Wiley \& Sons, Inc., 1984.

[11] E. Pasolli, F. Melgani, and M. Donelli, "Automatic analysis of GPR images: A pattern-recognition approach," IEEE Trans. Geosci. Remote Sens., vol. 47, no. 7, pp. 2206-2217, 2009.

[12] W. Sun, H. Tang, H. Chen, and G. Yu, "Research of unsupervised image change detection algorithm based on 2-d histogram," in Proc. Int. Conf. on Signal Process., 2010, pp. 686-689.

[13] S. Patra and L. Bruzzone, "A fast cluster-assumption based activelearning technique for classification of remote sensing images," IEEE Trans. Geosci. Remote Sens., vol. 49, no. 5, pp. 1617-1626, 2011.

[14] P. Sahoo and G. Arora, "A thresholding method based on twodimensional renyi's entropy," Pattern Recognition, vol. 37, no. 6, pp. 1149-1161, 2004.

[15] D. Pham, S. Dimov, and C. Nguyen, "Selection of k in k-means clustering," Proc. Institution of Mechanical Engineers, Part C: J. Mechanical Engineering Sci., vol. 219, no. 1, pp. 103-119, 2005. 